\title{
A Crystal Plasticity-based Study of the Relationship between Microstructure and Ultra-High-Cycle Fatigue Life in Nickel Titanium Alloys
}

\author{
John A. Moore ${ }^{\mathrm{a}, \mathrm{d}, *}$, Dana Frankel ${ }^{\mathrm{b}}$, Rajesh Prasannavenkatesan ${ }^{\mathrm{c}}$, August G. Domel ${ }^{\mathrm{e}}$, Gregory B. Olson ${ }^{\mathrm{b}}$, Wing Kam \\ $\mathrm{Liu}^{\mathrm{d}, *}$ \\ ${ }^{a}$ Lawrence Livermore National Laboratory, PO Box 808, Livermore, CA 94551, USA \\ ${ }^{b}$ Department of Materials Science and Engineering, Northwestern University, Evanston, IL 60208, USA \\ ${ }^{c}$ QuesTek Inovations LLC, 1820 Ridge Ave in Evanston, IL 60201, USA \\ ${ }^{d}$ Department of Mechanical Engineering, Northwestern University, Evanston, IL 60208, USA \\ ${ }^{e}$ School of Engineering and Applied Sciences, Harvard University, Cambridge, MA 02138, USA
}

\begin{abstract}
Nickel Titanium (NiTi) alloys are often used in biomedical devices where failure due to mechanical fatigue is common. For other alloy systems, computational models have proven an effective means of determining the relationship between microstructural features and fatigue life. This work will extend the subset of those models which were based on crystal plasticity to examine the relationship between microstructure and fatigue life in NiTi alloys. It will explore the interaction between a spherical inclusion and the material's free surface along with several NiTi microstructures reconstructed from 3D imaging. This work will determine the distance at which the free surface interacts with an inclusion and the effect of applied strain of surface-inclusion interaction. The effects of inclusion-inclusion interaction, matrix voiding, and matrix strengthening are explored and ranked with regards to their influence on fatigue life.
\end{abstract}

Keywords: Microstructures, Finite elements, Surface effects, Image-based modeling

\section{Introduction}

Nickel Titanium (NiTi) alloys are often used in biomedical devices such and stents $[1,2]$ and artificial heart valves [3]. Failure of such devices due to mechanical fatigue has caused negative clinical outcomes [4] and has potential to increase healthcare cost and patient trauma if device replacement is needed. More fatigue resistant NiTi alloys will help to mitigate these issues.

Quantitative understanding of NiTi microstructures' effect on fatigue performance will allow for the design of improved alloys at a more rapid pace than with trialand-error development approaches.

A computational model offers the ability to assess the sensitivity of fatigue life to microstructural parameters in a controlled manner. This work will perform such a study. It will explore the potency of generic microstructures to nucleate fatigue cracks in a NiTi system, and it will address microstructures specific to drawn NiTi

\footnotetext{
${ }^{*}$ Corresponding author

Email addresses: moore236@llnl.gov (John A. Moore) w-liu@northwestern. edu (Wing Kam Liu)
}

Preprint submitted to International Journal of Fatigue tubes which are used in many biomedical applications [1].

Specifically this work will focus on determining a plastic strain-based damage parameter around nonmetallic inclusions in a NiTi alloy using computational crystal plasticity (CP). Unlike phenomenological plasticity models that capture observed trends in material behavior based on macroscale experimental data, $\mathrm{CP}$ predicts anisotropic plastic deformation based on slip mechanisms in crystal systems.

A number of studies have used CP modeling to explore fatigue nucleation around inclusions. Parametric studies of microstructural parameters were performed by Shenoy et al. [5] using CP. They used a 2D finite element model to study the effects of inclusion aspect ratio, spacing, and distance to free surface on crack incubation life for nickel superalloys. Kumar et al. [6] studied $\mathrm{Ni}_{3} \mathrm{Al}$ precipitates in single crystal nickel superalloys. They used CP to model strain distributions around large arrays of square precipitates and showed the effect of precipitate volume fraction on fatigue driving force. Wang et al. [7] studied fatigue crack incubation for interacting inclusions in separate grains

April 22, 2016 
of an aluminum 7075 alloy. Their analysis used CP to study 3D geometries extruded from circular and elliptical inclusion geometries. Alley and Neu [8] used a combined austenite to martensite phase transformation and CP model to study 3D cuboid and cubic inclusions in a 41100 steel, showing the effect of retained austenite on a plastic strain-based damage parameter. Hochhalter et al. [9] used CP to study a number of nonlocal nucleation metrics around ellipsoidal inclusions in 7075-T651 aluminum; they also applied these metrics to cracked $\mathrm{Al}_{7} \mathrm{Cu}_{2} \mathrm{Fe}$ particles which were reconstructed from 2D images. Prasannavenkatesan et al. [10] used a 3D CP model to study the effects of hard $\mathrm{Al}_{2} \mathrm{O}_{3}$, soft $\mathrm{La}_{2} \mathrm{O}_{2} \mathrm{~S}$ and pores on fatigue life in martensitic gear steels. Salajegheh et al. [11] determined the probability of failure in the nickel superalloy IN100, using a 2D crystal plasticity model of $2 \mu \mathrm{m}$ inclusions. While all of these works studied fatigue crack nucleation around inclusions using CP, none focused specifically on NiTi.

Other crystal plasticity models, such as those of Wang et al. [12], Manchiraju and Anderson [13], Manchiraju et al. [14], Yu et al. [15, 16, 17, 18], and Paranjape and Anderson [19] have focused specifically on NiTi but did not study fatigue or inclusions ${ }^{1}$.

Two final studies of note are that of Przybyla et al. [20] and Salajegheh et al. [21]. Przybyla et al. [20] used a 2D model to determine a strain-based damage parameter as a function of surface distance to a hard inclusion in the presence of an oxide layer, which this work will parallel albeit in 3D and without considering surface oxidation. Salajegheh et al. [21] studied the behavior of a strain-based damage parameter with respect to interaction and orientation between inclusions in $2 \mathrm{D}$ and orientation between inclusions in 3D; the present work will address similar inclusion interactions based on a NiTi specific microstructure. However, both Przybyla et al. and Salajegheh et al. used an isotopic phenomenological plasticity model rather than $\mathrm{CP}$.

This work will, for the first time, use the microstructure-sensitive fatigue models developed for steels, aluminums, and nickel superalloys combined with CP models of NiTi to address the effects of nonmetallic inclusions on NiTi fatigue life.

This work will address the effect of inclusion-surface interactions, inclusion-inclusions interactions, inclusion morphology and an inclusion/matrix interface void on fatigue life. The results of these studies will then be

\footnotetext{
${ }^{1} \mathrm{~A}$ number of studies (see [18] for a review) model phase transformation in NiTi based on similar concepts to crystal plasticity, these are often called crystal plasticity models but do not consider plastic deformation specifically.
}

summarized in the contexts of materials design and fatigue resistance improvements for NiTi materials.

\section{Fatigue Prediction Method}

Cracks that will ultimately cause fatigue failure can form at a variety of microstructural features such as grain boundaries, surface defects and inclusions [22]. When samples fail at $10^{8}-10^{10}$ cycles [23], the failure process is often termed Ultra-High-Cycle Fatigue (UHCF). This scenario is of particular interest for biomedical applications which are frequently in continuous service for up to 20 years $^{2}$. Studies of SUJ2 steel $[25,26,27,28,29,30]$ have shown that sub-surface fatigue nucleation at inclusions becomes the dominant mode of nucleation when samples fail in (or near) the UHCF regime. Also, fatigue crack nucleation at inclusions is a common concern in biomedical materials [31]. For this reason, this work will focus only on inclusions.

The fatigue life of a material can be decomposed into several stages [32]:

$$
N=N_{\mathrm{inc}}+N_{\mathrm{MSC}}+N_{\mathrm{PSC}}+N_{\mathrm{LC}},
$$

where $N$ is the total fatigue life, $N_{\text {inc }}$ is the number of crack incubation cycles, $N_{\mathrm{MSC}}$ is the number of cycles of microstructurally small crack growth, $N_{\mathrm{PSC}}$ is the number of cycles of physically small crack growth, and $N_{\mathrm{LC}}$ is the number of cycles of long crack growth. Incubation refers to the number of cycles for crack nucleation and growth beyond the influence of the feature or defect were nucleation occurred. Microstructurally small and physically small crack growth are often lumped together into one stage of crack growth beyond microcrack influence but where linear elastic fracture mechanics is invalid [32].

This work will focus on characterizing the potency of NiTi microscale features to incubate fatigue cracks. Based on these, results the fatigue incubation life will be predicted. Lankford and Kusenberger observed that the variation in fatigue life of 4340 steels was dominated by crack initiation; furthermore, McDowell [22] observed that the fatigue life beyond the initial crack site is often an "inconsequential" fraction of fatigue life. Thus, the comparisons of $N_{\text {inc }}$ for various microstructures will be considered valid for total fatigue life as well.

\footnotetext{
${ }^{2}$ The normal resting heart rate for an adult is $60-100$ beats per minute [24]. A heart beating at 100 beats per minute for 20 years results in $1,051,898,000$ heart beats $(\approx 1$ billion). This represents the approximate service life of an artificial heart valve.
} 
This work will link fatigue incubation life to microscale simulation outputs through Goh and McDowell's [33] extension of Fatime and Socie's [34] "Fatigue Indicator Parameter" (FIP) given by:

$$
\frac{\Delta \gamma_{\max }^{p}}{2}\left(1+\kappa \frac{\sigma_{n}^{\max }}{\sigma_{y}}\right)=\tilde{\gamma}_{f}\left(2 N_{\mathrm{inc}}\right)^{c}=F I P,
$$

where $\Delta \gamma_{\max }^{p}$ is the change in maximum cyclic plastic shear strain averaged over a finite volume, and $\sigma_{n}^{\max }$ is the stress normal to the critical plane of $\gamma_{\max }^{p}$. The parameters $c$ and $\tilde{\gamma}_{f}$ are Coffin-Manson type calibration parameters and $\kappa$ is a constant determined to equate $F I P$ for uniaxial and torsional loaded specimens [34].

The values of $\Delta \gamma_{\max }^{p}$ and $\sigma_{n}^{\max }$ are determined from crystal plasticity-based finite element simulations of NiTi microstructures, from which FIP is calculated and averaged over a region $10 \%$ of the volume of an inclusion in accordance with the procedure in [35]. This volume averaging reduces mesh dependency and introduces a length scale into fatigue life calculations. The "fatigue indicator parameter" gages the potency of microstructural features to incubate fatigue cracks. Calibrating FIP values for a given microstructure to fatigue life data using $c$ and $\tilde{\gamma}_{f}$ establishes a baseline microstructure to which other microstructures can be compared. This work will use a microstructure based on 3D imaging as such a baseline.

For this study, volume averaging was performed over a cubic region. The edge length of this region has ranged from 1-3 $\mu \mathrm{m}$ in previous simulations of inclusions $[5,11,10]$, but Shenoy et al. suggests that 0.3-1 $\mu m$ should be a lower bound. This work uses an edge length of $0.23 \mu \mathrm{m}$, which is consistent with the procedure in [35], but just below Shenoy et al.'s suggested range. However, the $0.23 \mu \mathrm{m}$ window amounts to a cubic volume region with faces consisting of $5 \times 5$ element. Figure 9 shows that this is roughly the area of the interface between carbide and void, and thus, is good estimate of the length scale on which damage has been observed.

\section{Computational Micromechanics}

Computational crystal plasticity will act as the constitutive modeling framework for this study. Several versions of computational crystal plasticity exist. Each version differs slightly in its continuum mechanics, hardening laws, calibration methods and algorithmic details. This work will use the $\mathrm{CP}$ version of McGinty and McDowell $[36,37]$. This version employs an implicit time integration algorithm for both the material law and finite element method (FEM) kinematic variable updates. The details of this CP version are given in [36] but the basic assumptions of the approach will be discussed below.

The plastic deformation gradient $\boldsymbol{F}$, is multiplicatively decomposed into an elastic $\boldsymbol{F}^{e}$ and plastic $\boldsymbol{F}^{p}$ part as:

$$
\boldsymbol{F}=\boldsymbol{F}^{e} \cdot \boldsymbol{F}^{p},
$$

which maps a point in a reference configuration onto an intermediate configuration and then a current (i.e., fully deformed) configuration [38]. In Equation 3, $\boldsymbol{F}$ is known from the FEM and $\boldsymbol{F}^{p}$ is determined from the solution of the following ordinary differential equation:

$$
\dot{\boldsymbol{F}}^{p}=\tilde{\boldsymbol{L}}^{p} \cdot \boldsymbol{F}^{p},
$$

where $(\dot{\star})$ is the time derivative and $\tilde{\boldsymbol{L}}^{p}$ is the plastic velocity gradient in an intermediate configuration which accounts for plastic deformation of a material but not elastic deformation or rigid rotation (see [36] or [38]). In Equation $4, \tilde{\boldsymbol{L}}^{p}$ is given by:

$$
\tilde{\boldsymbol{L}}^{p}=\sum_{\alpha=1}^{N_{\text {slip }}} \dot{\gamma}^{(\alpha)}\left(\tilde{\boldsymbol{s}}^{(\alpha)} \otimes \tilde{\boldsymbol{m}}^{(\alpha)}\right),
$$

where $\otimes$ is the tensor product, $N_{\text {slip }}$ is the number of slip systems, $\dot{\gamma}^{(\alpha)}$ is a shear slip rate, $\tilde{\boldsymbol{s}}^{(\alpha)}$ is the slip direction, and $\tilde{\boldsymbol{m}}^{(\alpha)}$ is the slip plane normal, all for a crystal slip system $(\alpha)$ in the intermediate configuration. The shear slip rate is approximated with a power-law as:

$$
\dot{\gamma}^{(\alpha)}=\dot{\gamma}_{0}\left(\frac{\tau^{(\alpha)}-a^{(\alpha)}}{\tau_{0}^{(\alpha)}}\right)^{m},
$$

where $\tau^{(\alpha)}$ is the resolved shear stress, $a^{(\alpha)}$ is a backstress that describes kinematic hardening, $\dot{\gamma}_{0}$ is a reference shear strain rate, $\tau_{0}$ is a reference shear stress, and $m$ is a material parameter. The resolved shear stress is determined by :

$$
\tau^{(\alpha)}=\boldsymbol{\sigma}:\left(\boldsymbol{s}^{(\alpha)} \otimes \boldsymbol{m}^{(\alpha)}\right),
$$

where (:) is a tensor double contraction and $\boldsymbol{\sigma}, \boldsymbol{s}^{(\alpha)}$, and $\boldsymbol{m}^{(\alpha)}$ are the Cauchy stress, slip direction and slip plane normal respectively, all of which are in the current configuration. The reference shear stress $\tau_{0}$ accounts for isotropic hardening and is considered constant in time. The backstress $a^{(\alpha)}$ evolves based on the expression:

$$
\dot{a}^{(\alpha)}=h \dot{\gamma}^{(\alpha)}-r a\left|\dot{\gamma}^{(\alpha)}\right|
$$

where $h$ and $r$ are direct and dynamic hardening factors respectively. 
From the solution of Equation 4, the elastic deformation gradient is determined as, $\boldsymbol{F}^{e}=\boldsymbol{F} \cdot\left(\boldsymbol{F}^{p}\right)^{-1}$, from which the elastic Green-Lagrange strain $\boldsymbol{E}^{e}$ and Second Piola-Kirchhoff stress $\boldsymbol{S}^{e}$ are calculated. The Cauchy stress can then be updated using:

$$
\boldsymbol{\sigma}=\frac{1}{J^{e}}\left[\boldsymbol{F}^{e} \cdot \boldsymbol{S}^{e} \cdot\left(\boldsymbol{F}^{e}\right)^{T}\right],
$$

where $J^{e}$ is the determinate of $\boldsymbol{F}^{e}$. This stress update algorithm constitutes a set of non-linear equations for the increment in slip rates $\dot{\gamma}^{(\alpha)}$, which are solved iteratively.

\section{Material and Model Calibration}

\subsection{Material}

This work will consider an equiatomic NiTi alloy with a homogenous matrix crystal structure and nonmetallic inclusions. Plastic deformation is considered to occur in the B2 austenite phase of the NiTi matrix. In the austenite phase, six $\langle 100\rangle /\{110\}$ and six $\langle 100\rangle /\{010\}$ slip systems (as reported for a single crystal by [39] and used in a CP model by [13] ) will be considered.

It is of note that these systems do not produce five independent slip systems, and thus will not allow arbitrary deformations modes [40]. Thus, for large plastic deformations in a polycrystal-which will not be considered here-other slip systems or deformation mechanisms will come into play. The $\langle 100\rangle /\{110\}$ system is known to be the primary slip mode [41] [42]. The secondary system included in this work is $\langle 100\rangle /\{010\}$, which follows the CP study in [13] ; however, recent studies [41] have shown that $\langle 1 \overline{1} 1\rangle /\{110\}$ is more energetically favorable for dislocation glide than $\langle 100\rangle /\{010\}$. While $\langle 1 \overline{1} 1\rangle /\{110\}$ would allow for nine slip systems and arbitrary deformations, the six $\langle 100\rangle /\{010\}$ systems allow for higher strains in each system which is in line with worst-case scenarios analysis approach discussed in Subsection 4.3.

NiTi materials undergo a stress-assisted martensitic phase transformation between an ordered B2 austenite phase and a monoclinic B19' martensite phase [43, 44] when service temperature is above the material's austenite finish temperature $\left(A_{f}\right)$. Many NiTi alloys employed in biomedical applications have carefully tuned $A_{f}$ temperatures just below the service temperature (body temperature) such that the aforementioned phase transformation occurs. The result of this stress-assisted phase transformation in NiTi is the phenomenon of superelasticity (i.e., the material exhibits large recoverable transformation strains) [44] which is illustrated in Figure 1. Figure 1 shows schematics of two distinct NiTi responses in different temperature regimes. When the temperature $(T)$ is greater then $A_{f}$, the material is superelastic and when temperature is lower then the material's martensite finish temperature $\left(M_{f}\right)$ the material exhibits a shape-memory response. The superelastic material begins in a fully austenite state and nonlinearity is caused by a martensitic phase transformation, while the shape-memory material begins as martensite with several self-accommodated crystallographic variants and the nonlinearity is caused by a thermallyreversible reorientation of the variants along the direction of the applied load [45]. Also, the martensite can have an internally twinned structure of two twin related martensite variants (here called transformation twins), which can detwin upon further loading [46]. Thus, for a generic NiTi alloy, the plastic velocity gradient of Equation 5 should be replaced by an inelastic velocity gradient:

$$
\tilde{\boldsymbol{L}}^{\text {inel }}=v_{A} \tilde{\boldsymbol{L}}^{p}+\tilde{\boldsymbol{L}}^{\text {trans. }}+v_{M}\left(\tilde{\boldsymbol{L}}^{\mathrm{twin}}+\tilde{\boldsymbol{L}}^{\mathrm{RO}}+\tilde{\boldsymbol{L}}^{p M}\right),
$$

which accounts for deformation due to transformation $\left(\tilde{\boldsymbol{L}}^{\text {trans. }}\right)$, detwinning $\left(\tilde{\boldsymbol{L}}^{\text {twin }}\right)$, reorientation $\left(\tilde{\boldsymbol{L}}^{\mathrm{RO}}\right)$, and slip in the martensite $\left(\tilde{\boldsymbol{L}}^{p M}\right)$, weighted by the respective volume fractions of austenite $\left(v_{A}\right)$ and martensite $\left(v_{M}\right)$. However, the scope of this work will be limited to the case of UHCF for cyclic loads similar to those encountered in biomedical devices, where $\tilde{\boldsymbol{L}}^{\text {trans. }}, \tilde{\boldsymbol{L}}^{\text {twin }}, \tilde{\boldsymbol{L}}^{\mathrm{RO}}$, $\tilde{\boldsymbol{L}}^{p M}$ are negligible, as will be discussed in the remainder of this sub-section.

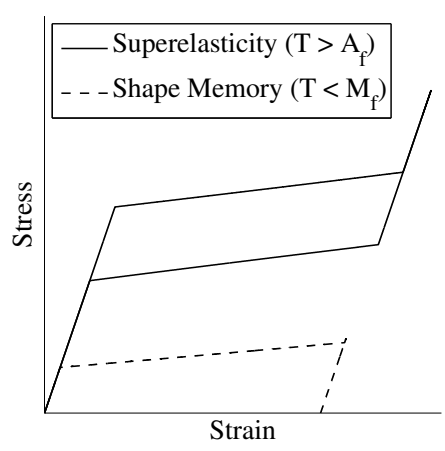

Figure 1: Example stress-strain curves for NiTi in two temperature regimes that result in superelastic and shape-memory behavior

The service life of deployed biomedical devices, such as stents, can be divided into three stages: initial crimp, deployment and in vivo cycles as discussed in [44]. The initial crimp stage is associated with the largest strain the device will undergo and is accompanied by an increase in martensite volume fraction. During the 
deployment stage, a partial unloading of strain occurs as the device expands, and the ensuing partial reverse martensitic transformation results in a reduction of the martensite volume fraction. During the in vivo cycle stage (where most of the fatigue life occurs), the change in strain levels are small compared to the crimp and deployment strains; it is in the in vivo cycle stage where microscale plastic deformation will be determined for this study.

While many have performed fundamental studies on the cyclic behavior of superelastic NiTi (Notably Miyazaki et al. [47, 48, 49] and Gall et al. [46, 50, 51]), these studies have not addressed the unique nature of the initial crimp and in vivo cycles typical of stent devices, focusing instead on large cyclic strain - relative to the in vivo cycles - in the absence of any initial pre-strain. Pelton [52], however, focused specifically on the behavior of superelastic NiTi under initial crimp and in vivo cyclic conditions, showing that the microstructure of annealed NiTi samples after 10 simulated in vivo cycles is comprised of dislocation rich austenite "interspersed" with retained martensite [52].

Pelton [52] suggests that, under stent loading conditions, the motion of these martensite interfaces causes interfacial dislocations which, upon further transformation cycling, become "tangled, shorter, and more curved" and subsequently impede further motion of the martensite interface. Based on this observation, the present work will assume that martensite interfaces are stationary during in vivo cycling. This assumption is illustrated by the stress-strain curves in Figure 3a of Pelton's work [52] where the hysteresis due to transformation and slip decreases over time leaving what appears to be only hystereses due to plasticity as indicated by the continued increase in mean strain after many cycles. Kim and Miyazaki [53, 54] also show that under UHCF conditions the macroscale strain at failure is less than that needed for transformation. While Kim and Miyazaki's data addresses only macroscale strain, it confirms the trend that transformation strain decreases when strains approach UHCF conditions. Thus, while there will be significant transformation strain during initial crimp, $\tilde{\boldsymbol{L}}^{\text {trans. }}$ will be insignificant during in vivo cycling, and will not contribute to the "Fatigue Indicator Parameter" for high or ultra-high-cycle in vivo conditions.

As a note, the transformation strains are associated with transformations twins [45] which are internal to the martensite phase. Thus, the aforementioned interfacial dislocations may occur, in part, due to the presence of these twins. However, the logic presented to assume
$\tilde{\boldsymbol{L}}^{\text {trans. }}=0$ during in vivo cycling is assumed to hold regardless off the dislocation's source. It has also been shown by Norfleet et al. [55] that these transformationtwin-induced dislocations occur in the same slip systems as the austenite. Based on this observation, it will be assumed that any interaction between glide dislocations and transformation twins does not notably change the plastic behavior of the austenite (the dual phase nature of the material is discussed in more detail below).

Adler et al. discussed in [54] that continued cycling of a NiTi work hardens the martensite phase and promotes reversible deformation mechanisms such as detwinning. It will be assumed that any detwinning during ultra-high-cycle in vivo cycling will result in a negligible number of irreversible dislocations or crack nucleating features. Therefore, the contribution of detwinning strain to the "Fatigue Indicator Parameter" will also be negligible. Due to this assumption, $\tilde{\boldsymbol{L}}^{\text {twin }}$ will be set to zero during simulations of the in vivo cycling.

For the $T>A_{f}$ curve in Figure 1, fully-oriented martensite will form during the crimp stage and remain until it is transformed back to austenite upon unloading; the martensite will not reorient unless there is a change in load direction or stress state [46]. Since, the change in plastic strain during in vivo cycling is small, the stress state (i.e., the relative magnitude of stresses around the inclusions) will remain constant, no reorientation will occur, and $\tilde{\boldsymbol{L}}^{\mathrm{RO}}$ will be considered negligible.

Finally, as shown in Sehitoglu et al [56], the austenite plastic yield stress is approximately $40 \%$ of that of the martensite. During in vivo cycling, plastic strains will be low (indicating marginal excedence of the B2 yield strength) thus, the B2 plastic flow behavior will dominate and $\tilde{\boldsymbol{L}}^{p M}$ will not be considered.

To address the effects of the crimping and deployment stages in the study of the in vivo cycle stage, the CP model was calibrated with the stress-strain data from Gall et al. [51] for a monotonically loaded colddrawn NiTi specimen in the "as received" condition. The cold-drawn "as received" sample was chosen because it exhibited only elasto-plastic deformation and was not observed to undergo an austenite to martensite phase transformation during loading, similar to the lack phase transformation encountered in the in vivo cycle stage. This sample also contained a mixture of austenite and martensite due to the cold-drawing process, which is similar to the aforementioned dual phase microstructure observed after 10 in vivo cycles in Pelton's [52] work. Thus, the CP model was calibrated to the elastic behavior of the dual phase material and the plastic behavior of the B2 austenite phase. 
The above assumptions allow the $\mathrm{CP}$ model to focus on the plastic behavior driving the fatigue process while avoiding the complexities of the unknown dual phase morphology induced by the crimping and deployment stages of deployed biomedical devices.

\subsection{Calibration of Crystal Plasticity Model}

To calibrate the CP model, a statistical volume element (SVE), where the material response differs from the macroscale homogeneous response, was considered and calibrated with the stress-strain data from Gall et al. [51] discussed in the previous sub-section. This SVE considered 27 crystallographic grains with constant crystal structures that were randomly sampled (in accordance with the procedure in [36]) 64 times. The SVE mesh is shown in Figure 2. Based on these SVE

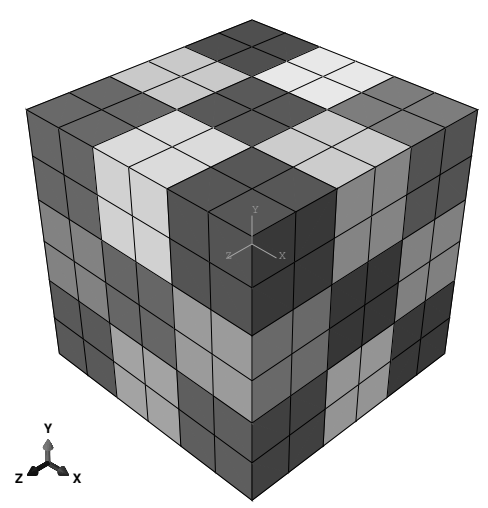

Figure 2: Finite element mesh of SVE with 27 grains. Colors (grayscale) represent grains

results, the stiffness tensor components $C_{1111}, C_{1122}$, $C_{2323}$, the constant reference slip rate $\dot{\gamma}_{0}$, the constant power law exponent $m$, and the reference shear stress $\tau_{0}$ were determined and are given in Table 1.

\begin{tabular}{c|c|c|c|c|c}
$C_{1111}, \mathrm{MPa}$ & $C_{1122}, \mathrm{MPa}$ & $C_{2323}, \mathrm{MPa}$ & $\dot{\gamma}_{0} \mathrm{~s}^{-1}$ & $m$ & $\tau_{0}, \mathrm{MPa}$ \\
\hline 80869 & 40356 & 20257 & 0.002 & 10 & 320
\end{tabular}

Table 1: Crystal plasticity material parameters for B2 NiTi

\subsection{Calibration of Fatigue Indicator Parameter}

With a focus on biomedical applications, UHCF will be considered. Also, in biomedical applications (where the cost of failure is assumed to be high) it is desirable to determine the fatigue life with a minimum probability of failure for a given microstructural configuration. To achieve this, the crystallographic orientation most conducive to plasticity will be modeled and calibrated to the minimum fatigue lives estimated from fatigue data. This work will use minimum fatigue life estimates based on the NiTi fatigue data from Pelton et al. [2] for diamondshaped-strut NiTi specimens. To determine the minimum life, the data was normalized so that it was independent of applied strain level. This was achieved by applying the following linear transformation as outlined by Harlow [57]:

$$
Z^{j}=a N+b,
$$

where $Z^{j}$ is the normalized fatigue life, $a=\frac{s^{j}}{s^{i}}$, and $b=\bar{N}^{j}-\frac{s^{j}}{s^{i}} \bar{N}^{i}$. Here $\bar{N}^{i}$ and $s^{i}$ are the respective mean and standard deviation of the untransformed data at a given strain level and $\bar{N}^{j}$ and $s^{j}$ the respective mean and standard deviation of the normalized data at a given strain level. For this analysis, both mean and standard deviation of the normalized data have been defined as 1 (i.e., $\bar{N}^{j} \equiv 1, s^{j} \equiv 1$ ). The normalized $Z^{j}$ data points were then fit to a 3-parameter Weibull distribution function. The 3-parameter Weibull distribution is given by:

$$
P\left(Z^{j}\right)=1-\exp \left[\left(\frac{Z^{j}-\gamma}{\beta}\right)^{\alpha}\right]
$$

where $P$ is the probability of failure, and $\alpha$ and $\beta$ are the shape and scale factors of the distribution respectively. In Equation 12, $\gamma$ is the normalized fatigue life with zero probability of failure. The probability of failure is determined from the data by rank ordering values of $Z^{j}$ in increasing order of magnitude with ranks numbering from $j=1$ to $j=\mathscr{M}$ :

$$
P\left(Z^{j}\right)=\frac{j}{\mathscr{M}+1},
$$

where $\mathscr{M}$ is the number of fatigue life data points at a given strain. Because Pelton did not differentiate surface crack nucleation (here referred to as Mode $A$ failure) from bulk/sub-surface crack nucleation (Mode $B$ failure) data, some assumptions were made to determine the Mode $B$ minimum fatigue life in the UHCF region.

To determine the minimum fatigue lives from Pelton's NiTi fatigue data, it was assumed that the ratio of Mode B to Mode A mean and standard deviation values (at the Mode A fatigue threshold) was constant for all materials. It was likewise assumed that the slope of the power law (Coffin-Manson) curve and values of $\alpha$ and $\beta$ that are used to model Mode $B$ minimum life behavior are also constant for all material systems. Using these assumptions, similar statistical analysis performed on SUJ2 bearing steel data was used to make predictions about NiTi Mode $B$ fatigue behavior.

The SUJ2 Mode A and Mode B data was consolidated from $[25,26,27,29,30]$. The estimated Mode 
$B$ zero probability of failure strain $(\varepsilon)$-life curve is given by $\varepsilon=1.7951 N^{-0.091}$. The values of $\tilde{\gamma}_{f}$ and $c$ in Equation 2 are used to calibrate the fatigue indicator parameter from the worst case microstructural model to this curve. Calibrating FIP in the manner allows the model to predict the extent to which microstructural changes improve the zero probability of failure fatigue life of the material.

\section{Finite Element Modeling}

Two sets of finite element meshes will be used for this study: an ideal inclusion near a free surface and a pair of oxicarbide inclusions separated by a void and reconstructed from microscale imaging, both which will be discussed in their respective sections.

As a precursor to these studies, the number of cycles needed for saturation of $\Delta \gamma^{p}$ in Equation 2 was first considered. A single 8 node hexahedral finite element was loaded for eight cycles with $R=-1$, where $R$ is the ratio of minimum to maximum applied stress. Figure 3 shows that $\Delta \gamma^{p}$ saturates after 2-3 cycles. Three cycles will be used for the forthcoming study. In addition to the parameters in Table 1, the material parameters in Table 2 were used for all simulations unless otherwise noted. The resulting values of $\Delta \gamma^{p}$ are shown in Figure 3.

\begin{tabular}{c|c|c}
$a^{t=0}, \mathrm{MPa}^{*}$ & $h, \mathrm{MPa}$ & $r, \mathrm{MPa}$ \\
\hline 0.0 & 500 & 0 \\
\hline${ }^{*}$ initial backstress
\end{tabular}

Table 2: Material parameters used for finite element simulations

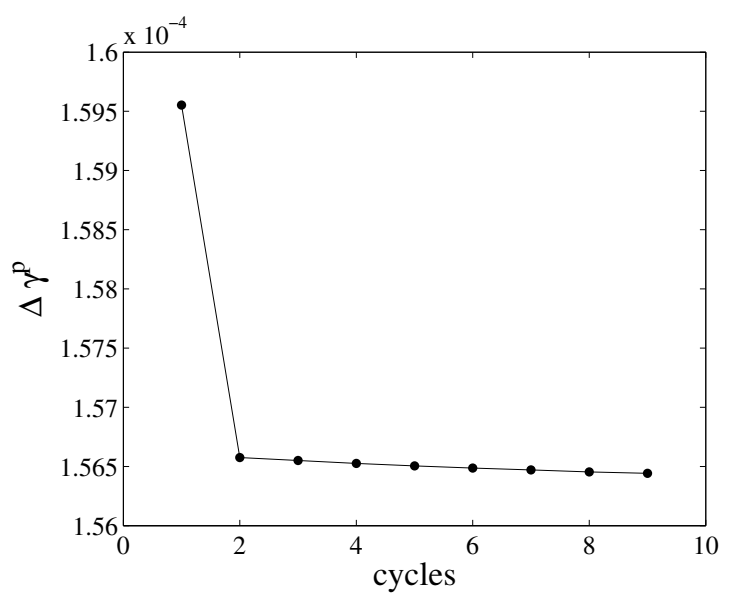

Figure 3: Saturation of $\Delta \gamma^{p}$ for a single element

For all forthcoming simulations, the volume averaging window, over which Equation 2 is calculated, will be set to $10 \%$ of the volume of the largest inclusion. Likewise, the maximum plastic strain increment $\Delta \gamma_{\max }^{p}$ in Equation 2 is determined based on the difference in plastic strains between two adjacent cycles. This value is measured at the maximum applied positive strain load in each cycle. The value is calculated as [58],

$$
\gamma_{\max }^{p}=\max _{\theta=1 \ldots N_{\theta}}\left[n_{i}^{\theta} \varepsilon_{i j}^{p} t_{j}^{\theta}\right]
$$

where $\boldsymbol{n}$ and $\boldsymbol{t}$ are normal and tangent vectors to a plane, $\theta$, respectively, which is iterated through $N_{\theta}$ values, and $\boldsymbol{\varepsilon}^{p}$ is the plastic strain tensor. The maximum stress in this plane $\left(\theta_{\max }\right)$ is a function of the Cauchy stress $\sigma$ and is determined by:

$$
\sigma_{\max }^{n}=n_{i}^{\theta_{\max }} \sigma_{i j} n_{j}^{\theta_{\max }} .
$$

\section{Study of Surface Effects}

As discussed in the previous section, fatigue cracks may nucleate at free surfaces or in the bulk material and nucleation at inclusions may occur far into the material or near the surface. Inclusions near the surface can be particularly potent for fatigue crack incubation, in part, due to a mechanical interaction between the surface and sub-surface inclusions. Even an ideal (flawless) surface will interact with sub-surface inclusions. This interaction will be detrimental to the fatigue life of the material. This section will determine the distance at which the surface and inclusions interact for a NiTi material under UHCF strain loadings and study effects of applied strain on surface-inclusion interaction.

To determine the effects of surface-inclusion interaction on fatigue life, several finite element meshes where generated with varying surface-to-inclusion distances. An example mesh is shown in Figure 4. These meshes ranged from 48000-59000 elements and 52000-64000 nodes. Eight node hexahedral elements were used exclusively. The displacement on the faces with normals in the negative directions were fixed in $x-, y$, and, $z-$ directions respectively. Each of the free edges was held flat using constraint equations.

Two load cases were considered. The first load case loaded the material with a displacement in the $z$ direction. This load case was meant to approximate either a uniaxial loading of a smooth specimen or a pressure loading on a thin walled tube specimen (i.e., hoop stress). The second load case loaded the material with a displacement in the $y$-direction, perpendicular to the free surface. This load case was meant to approximate the stress state in a more complex structure where the 


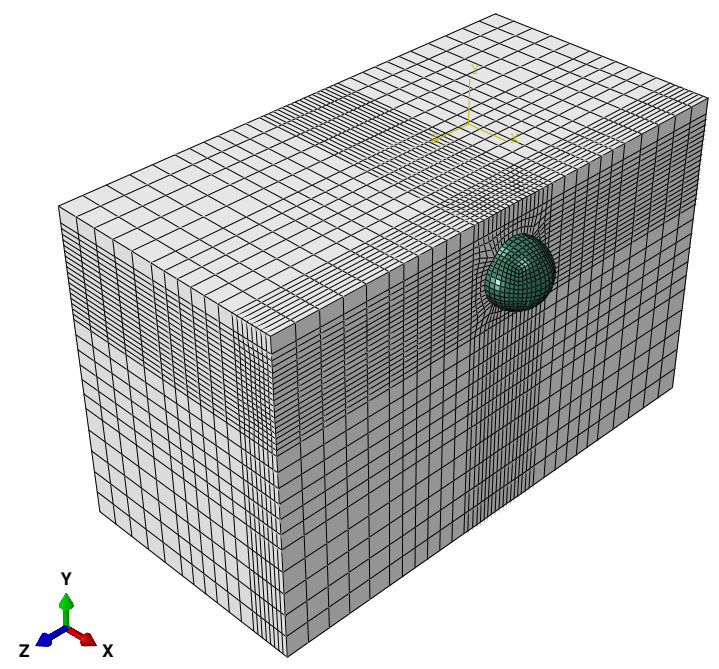

Figure 4: Example mesh with spherical inclusion near surface (top $y$-face). Only half of the matrix is shown to highlight the inclusion.

dominate stress is perpendicular to the surface. For all load cases, $R=-1$.

For loads in the $z$-direction, the crystal systems were rotated by Euler angles $\Psi=0^{\circ}, \theta=45^{\circ}, \phi=0^{\circ}$ (using the Roe convention) from a coordinate system aligned with the global axes and for loads in the $y$-direction, the crystal systems were rotated by Euler angles $\Psi=90^{\circ}$, $\theta=45^{\circ}, \phi=0^{\circ}$. These orientations are the most conducive to plastic slip in the (100)[001] system and thus are considered the worst-case scenarios consistent with the Mode B zero probability of failure analysis discussed in Section 4.

Pelton et al.'s [2] fatigue life data for NiTi suggests a traditional high-cycle fatigue endurance limit at approximately $0.5 \%$ strain. While our zero probability of failure curve $\left(\varepsilon=1.7951 N^{-0.091}\right)$ shows how UHCF failure may extend to lower strains, $0.5 \%$ strain will be consisted the maximum UHCF strain for this study. As will be shown (see Figure 6), the range at which the free surface affects fatigue life decreases with decreasing strain and thus studying $0.5 \%$ will give an estimate of the maximum reach of surface effects for UHCF.

The FIP values for both the $y$-and $z$-loadings are shown in Figure 5. In this figure, the values of FIP have been averaged over a window of elements approximately equal to $10 \%$ of the volume of the inclusion. As the inclusion is moved away from the surface, the volume where peak FIP occurs changes slightly. Due to the shape of the elements, the volume of each window is not exactly $10 \%$ of the volume of the inclusion

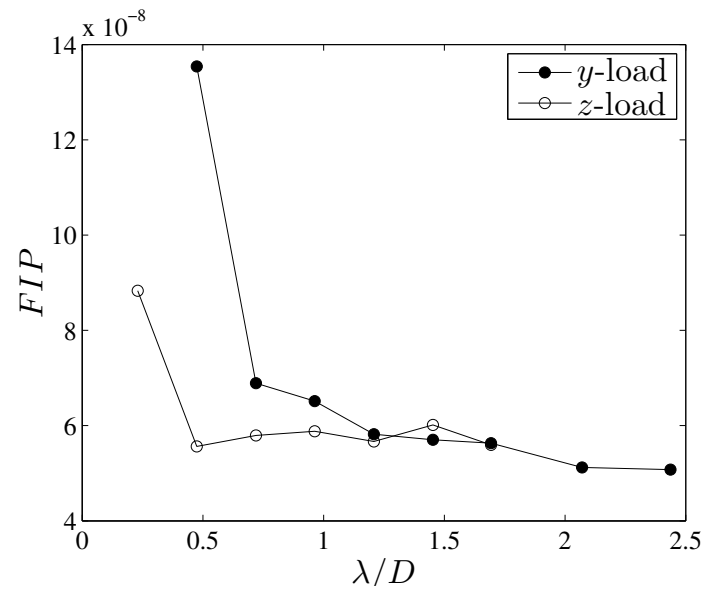

Figure 5: FIP as a function of the distance from the inclusion to the surface $\lambda$ normalized by the inclusion diameter $D$. A strain of $0.5 \%$ is applied in the respective directions.

which accounts, in part, for the lack of smoothness in each curve. Salajegheh et al. [21] showed a similar lack of smoothness due to mesh coarseness for their 3D calculations but found that it did not affect the overall trends of their predictions.

From Figure 5, it can be observed that the FIP values begin to converge at approximately 1.2 diameters from the surface. Thus, inclusions less than 1.2 diameters from the surface will have an increased potency to incubate fatigue cracks for applied strains of $0.5 \%$ or less. An example of fatigue failure for a $5.5 \mu \mathrm{m}$ carbide inclusion within the predicted range of the surface is shown in Figure 8 of [59].

Figure 6 shows that the distance at which surfaceinclusion interaction occurs decreases with decreasing strain. The star symbols in Figure 6 illustrate this by marking the approximate distance where interaction becomes minimal. Figure 7 shows that the ratio of the peak value $\left(F I P_{\text {peak }}\right)$ near the surface to the bulk value $\left(F I P_{0}\right)$ decreases as strain increases. This occurs because the stress concentration factor $K_{t}$ (the ratio of peak equivalent stress to applied stress) reduces more rapidly near the surface than in the bulk material as strain increased, as is illustrated in Figure 8. Thus, the elastic interaction between the surface and the inclusion decreases at higher strains.

Figure 8 shows that when applied strain is low, $K_{t}$ remains nearly constant. For a material with a plastic plateau in its stress-strain curve, $K_{t}$ should be constant when the matrix remains elastic then reduce and eventuality saturate to a lower value as the matrix transitions to a fully yielded state. This is shown in Figure 8 as $K_{t}$ reduces with increase applied strain as a result of plas- 


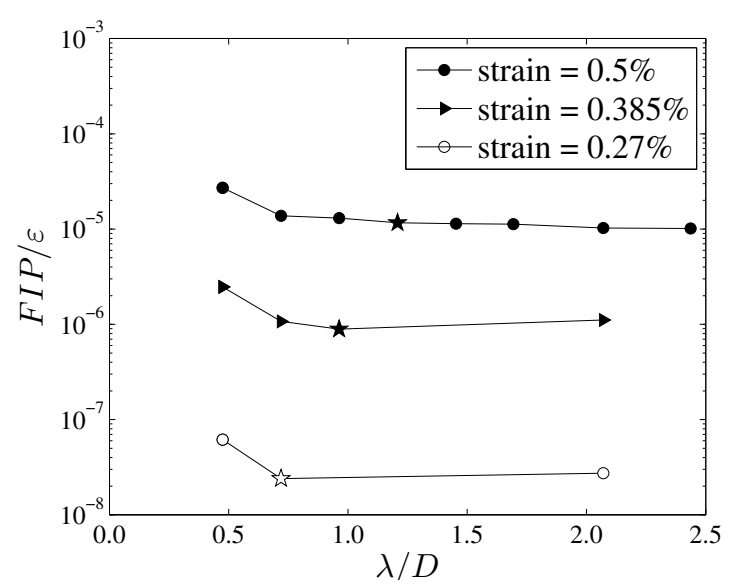

Figure 6: FIP normalized by applied strain $(\varepsilon)$ as a function of the distance from the inclusion to the surface $\lambda$ normalized by the inclusion diameter $D$ for loadings in the $y$-direction. The star symbols represent the approximate distance where surface-inclusion interaction becomes minimal.

ticity. Since the values of $K_{t}$ near the surface decrease more rapidly than in the bulk, the ratio of peak to bulk FIP decreases as well, as is shown in Figure 7. Thus, the increase in fatigue incubation potency of sub-surface inclusions as compared to bulk inclusions is amplified in the UHCF regime, and lowering strain should increase the probability of finding a critical inclusion (which will lead to failure) in the sub-surface rather than in the bulk material.

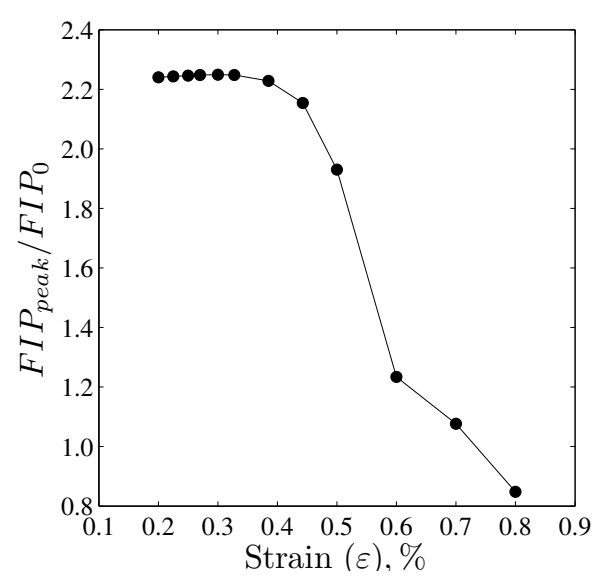

Figure 7: The peak fatigue indicator parameter near the surface $\left(F I P_{\text {peak }}\right)$ normalized by the value $F I P_{0}$ far from the surface for loadings in the $y$-direction. The plane $\theta$ used for calculating $\Delta \gamma^{p}$ is held constant.

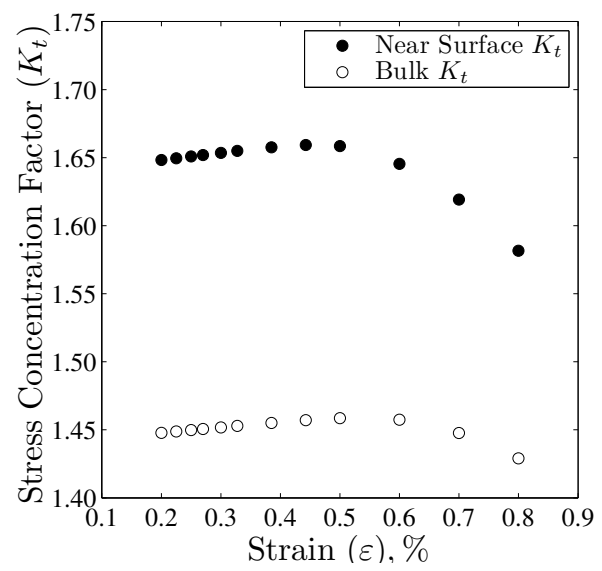

Figure 8: Stress concentration factors for an inclusion near the surface and far from surface (i.e., in the bulk material). The loading is in the $y$-direction.

\section{3D image-based analysis}

To further identify scenarios detrimental to fatigue life, an analysis was performed on a $3 \mathrm{D}$ reconstructed stringer of non-metallic oxicarbide $\operatorname{Ti}(\mathrm{C}, \mathrm{O})$ inclusions based on 3D imaging. Serial sectioning of NiTi tubes using a dual Focused Ion Beam (FIB)/Scanning Electron Microscope (SEM) was performed. The resulting $2 \mathrm{D}$ sections/images were reconstructed into a $150 \times 150$ $\times 1503 \mathrm{D}$ voxel image. Each voxel was designated as either NiTi matrix, inclusion or void. The small and large inclusions modeled were approximately $0.3 \mu \mathrm{m} \times$ $0.4 \mu \mathrm{m} \times 0.6 \mu \mathrm{m}$ and $0.6 \mu \mathrm{m} \times 0.6 \mu \mathrm{m} \times 1.0 \mu \mathrm{m}$ respectively. The inclusions are separated by approximately $0.6 \mu \mathrm{m}$ and connected by a void. The reconstructed inclusions are considered typical for drawn NiTi material.

To reduce computational cost, the initial voxel image was reduced to a $20 \times 20 \times 60$ voxel size. The reduced reconstruction is shown in Figure 9, where the void is modeled by deleting voided elements.

The reconstructed mesh was expanded to eliminate surface effects. Based on analysis in Section 6, each face was extended 1.2 times the major diameter of the largest inclusion. This mesh is shown in Figure 10.

Three meshes were created based on the mesh shown in Figure 10. These meshes modeled two inclusions with a void, two inclusions without a void, and one inclusion (the largest) without a void. Each of these meshes had 83228 elements and 86953 nodes (before element deletion). Eight node hexahedral elements were used exclusively. The displacement on the faces with normals in the negative directions were fixed in the $x$-, $y$ - and $z$-directions respectively. Each of the free edges 


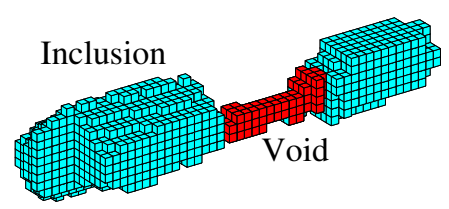

Figure 9: Reduced voxel representation of two oxicarbide inclusions and intermittent void

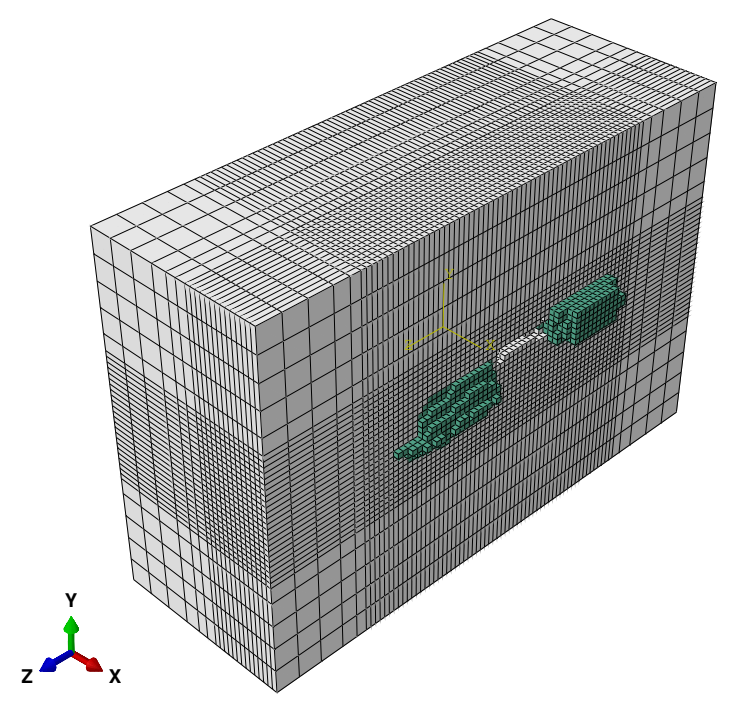

Figure 10: Reconstructed mesh of two oxicarbide inclusions with an intermittent void. The void is represented by the deleted elements shown between the inclusions. Only half of the matrix is shown to highlight the inclusions and void.

was held flat using constraint equations. The material parameters used in each simulation are shown in Tables 1 and 2. The displacement loading was applied in the $y$-direction and $z$-direction with $R=-1$. The worst case orientation was determined to be Euler angles $\Psi=90^{\circ}$, $\theta=40^{\circ}, \phi=5^{\circ}$.

The FIP results using the mesh with two inclusions and a void were determined and calibrated to the Mode $B$ zero probability of failure strain-life curve using $\tilde{\gamma}_{f}$ and $c$ in Equation 2. These values where calibrated by minimizing the logarithm of the root-mean-square error between predicted and experimental fatigue life, which yielded values $\tilde{\gamma}_{f}=0.0050$ and $c=-0.8195$. The calibrated strain-life curve is compared to the Mode B zero probability of failure strain-life curve in Figure 11.

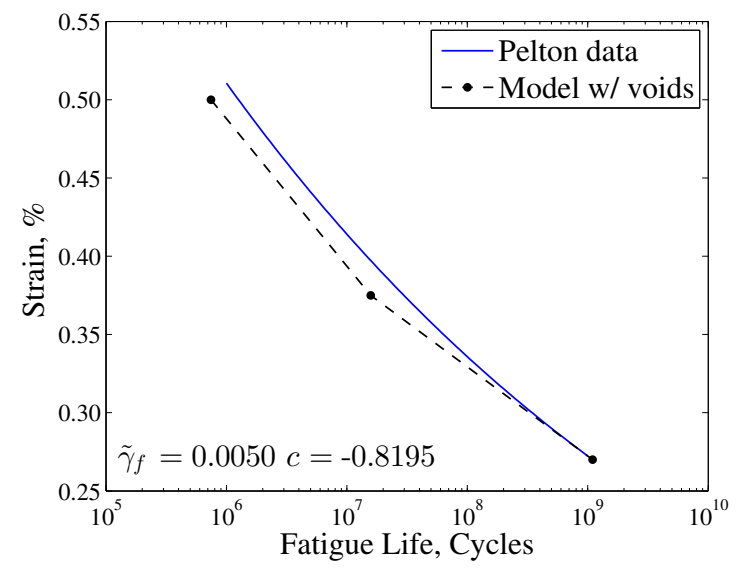

Figure 11: Calibration of FIP with Mode B zero probability of failure strain-life curve (determined from Pelton et al.'s data [2]) for a mesh with two inclusions and a void

Out of the three reconstructed microstructures studied (single oxicarbide, two oxicarbides without a void, and two oxicarbides with void), the case with two inclusions and a void will yield the highest $F I P$ values and thus the shortest fatigue life. For this reason, the case with two inclusions and a void were calibrated to the zero probability of failure fatigue lives. This calibration assumes that fatigue incubation life $\left(N_{\mathrm{inc}}\right)$ is approximately equal to the entire fatigue life $(N)$.

Using this calibration, the effects of microstructural features on fatigue life can be shown. Figure 12 shows the effect of interacting inclusions; Figure 13 shows the effect of the voiding between the inclusions created, most-likely, during the tube drawing process. Figure 14 shows the effects of matrix strengthening for two inclusions with a void, and Figure 15 compares the respective increases in fatigue life for all microstructures.

Salajegheh et al.'s [21] study of 2D round inclusions in an isotropic material found that FIP intensification due to inclusion-inclusion interaction was negligible when inclusion clusters were loaded parallel to the vector between their centroids and significant within distances on the order of one inclusion diameter when loaded perpendicular to this vector. The results in Figure 12 agree with this result for z-direction loading; however, for $y$-direction loadings, Salajegheh et al.'s results would suggest that a larger intensification of FIP should be observed (the inclusion spacing modeled here is $60 \%$ of the largest inclusions major diameter). It is unclear whether the inclusion morphology, dimensionality or anisotropy is driving this discrepancy. However, some conclusions can be drawn based on the analysis in Section 6. In the study of surface effects shown in Sec- 

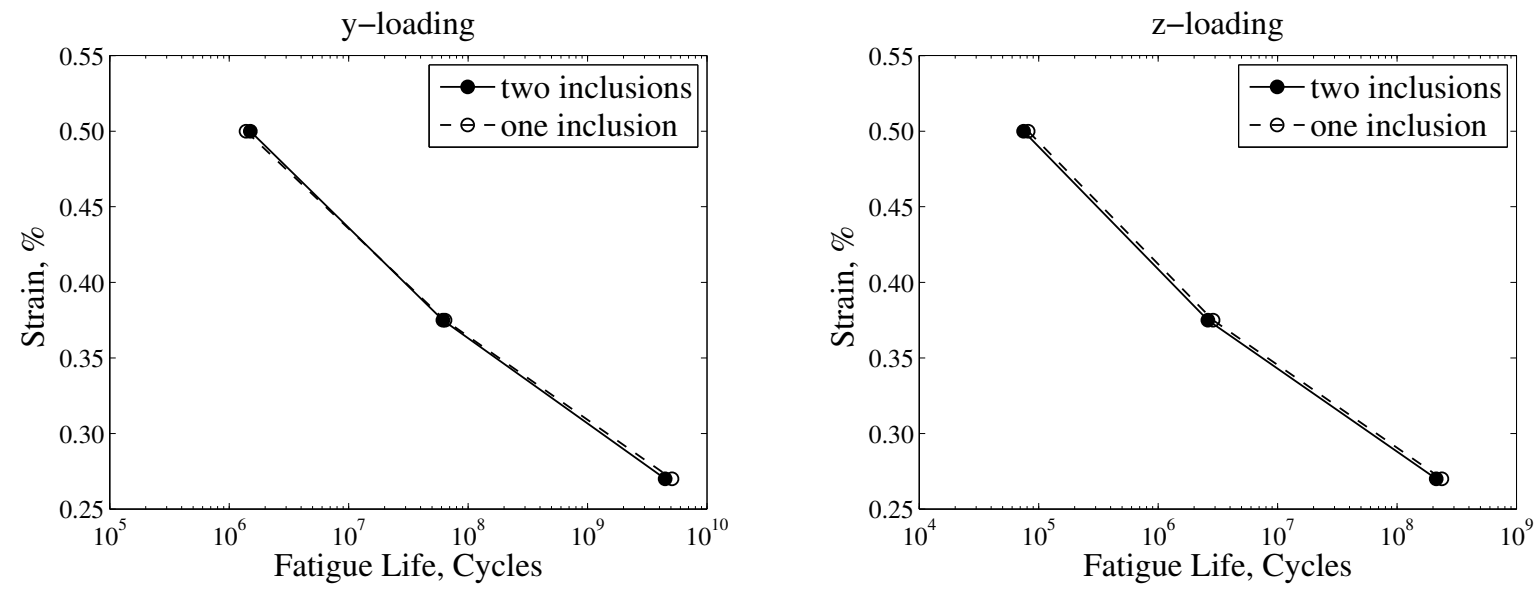

Figure 12: A comparison of fatigue life with one and two inclusions (and without void) for $y$ - and $z$-direction uniaxial loadings.
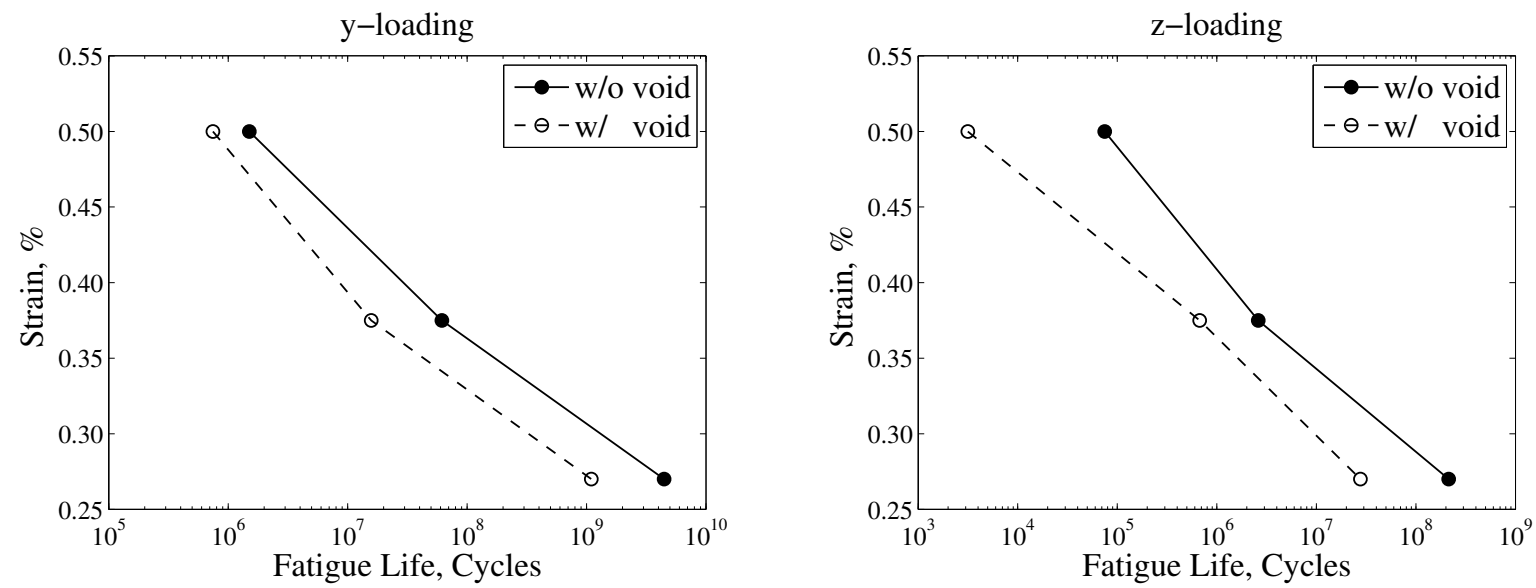

Figure 13: A comparison of fatigue life for two inclusions with and without the intermittent void for $y$-and $z$-direction uniaxial loadings.

tion 6, the maximum depth at which the surface played a mechanical role in UHCF crack incubation was determined to be 1.2 inclusion diameters. For an isotropic material, the boundary conditions would have yielded a model for two symmetric inclusions. Even though the NiTi material is anisotropic, the parallels to a symmetric system with two inclusions can be used to estimate the cutoff distance at which inclusion-inclusion interactions might affect fatigue life (i.e., twice the depth at which surface effects dissipated). Thus, two spherical inclusion could increase fatigue incubation potency when within 2.4 inclusion diameters of each other. However, this is not observed in the $3 \mathrm{D}$ reconstructed study. Based on this observation, it is concluded that the reconstructed morphology (i.e., the shape of the inclusion) is a more potent fatigue crack nucleant than the interaction between typical inclusions, and typical versus ideal inclusion morphology may be the major difference be- tween Salajegheh et al.'s and this study's results.

For a qualitative comparison with experimental fatigue data, Robertson et al.'s [31] data was considered, with the goal of comparing experimental data and model results for alloys with different inclusion stringer lengths.

Robertson et al. measured the fatigue life of a $\mathrm{NiTi}$ alloy under several processing conditions, included two alloys produced by vacuum arc remelting (VAR). Of these alloys, the High-Purity VAR (HP-VAR) processed material exhibited smaller and fewer inclusions than the standard-grade VAR (SG-VAR) alloy. Likewise, two models: one with a short inclusion stringer (one inclusion), and one with a long stringer (two inclusions with void) are compared to data for HP-VAR and SG-VAR respectively.

Robertson et al. provides probability of failure distributions at several fatigue lives for wire specimens. The 

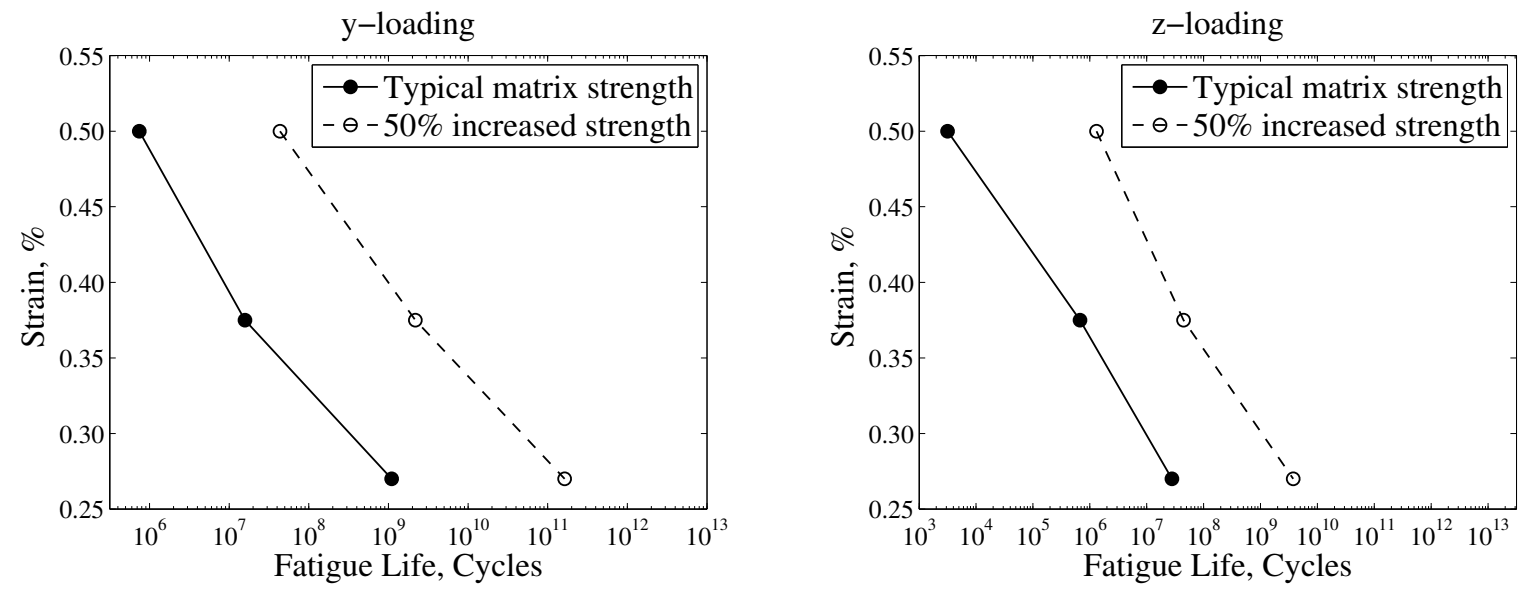

Figure 14: A comparison of fatigue life between a NiTi matrix with a typical yield strength and a NiTi matrix with a 50\% increased yield strength for $y$ - and $z$-direction uniaxial loadings.

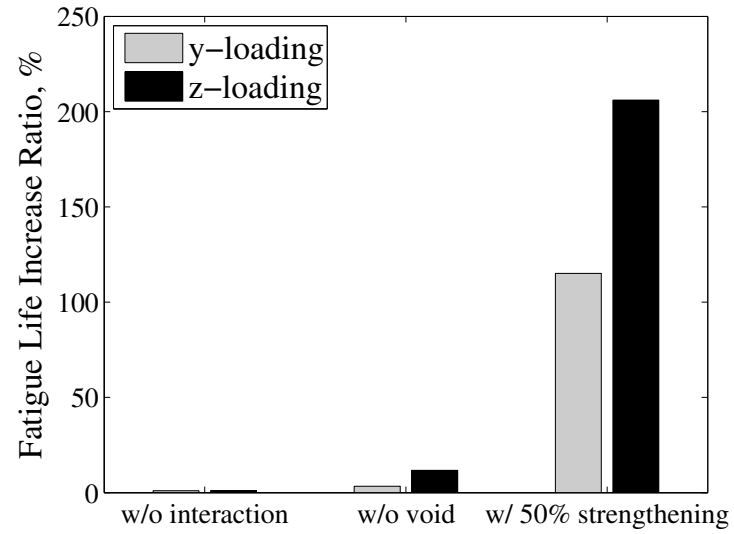

Figure 15: A comparison of the increase in fatigue life (i.e., the ratio of improved to original fatigue life) due to removing inclusion interaction, removing the void and increasing the NiTi matrix strength by $50 \%$.

model's short and long inclusion predictions are compared to $1 \%$ probability of failure data in Figure 16. One percent data was the minimum percentage distinguishable from Robertson et al.'s probability of failure distributions and is compared to model results in the $z$-direction to approximate the loading condition in the wire drawing direction.

The comparison in Figure 16 is qualitative only, because the mean inclusion length of the long and short stringers in the SG-VAR and HP-VAR respectively differed by a ratio of $3.66: 1$ whereas the two models showed a difference of 1.23:1, and the tested alloys showed differences in inclusion density not accounted for in the model. However, some information can be

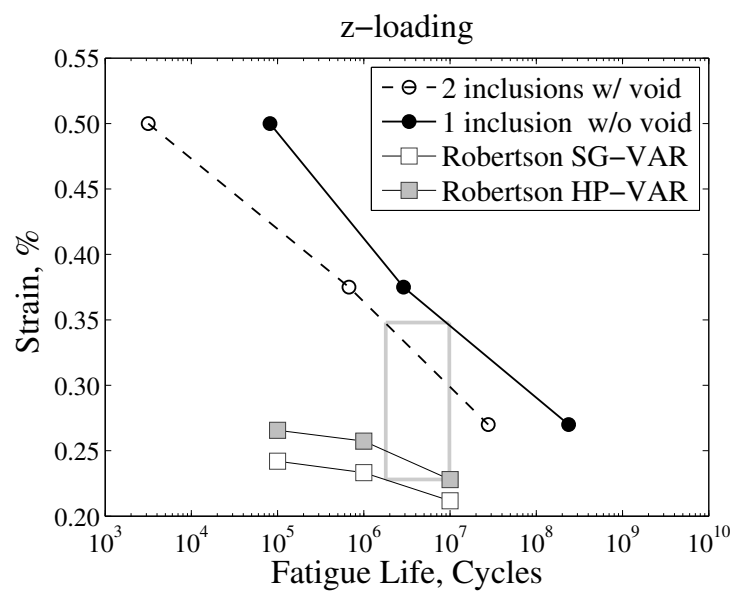

Figure 16: Predicted fatigue life for a short stringer (one inclusion) and a long stringer (two inclusions with void) compared to Robertson et al.'s [31] $1 \%$ probability of failure data for standard-grade and highpurity VAR wire samples. The gray rectangle highlights a life range were the model and data predict similar life increases.

taken from Figure 16. The most notable observation is that the model does not predict the strain for a given life correctly, but gives a more accurate estimate of the increase in life. Both the model and the data show an increase in life of slightly more than one order of magnitude for higher strains and slightly less than one order of magnitude for smaller strains. This illustrates the utility of this type of model, in that, the model gives a more accurate assessment of fatigue life improvements due to microstructures changes than of exact strain/life values.

Figure 17 shows a comparison between SEM images of fatigue cracks in NiTi at carbide/void interfaces 
(from Ramin et al. [60]) and the location of peak FIP values predicted by the model of two inclusions and a void. The figure illustrates that the model predicts regions with a high potency for crack nucleation at the inclusion/void interface and that this region's location agrees with the location of experimentally observed fatigue cracks.

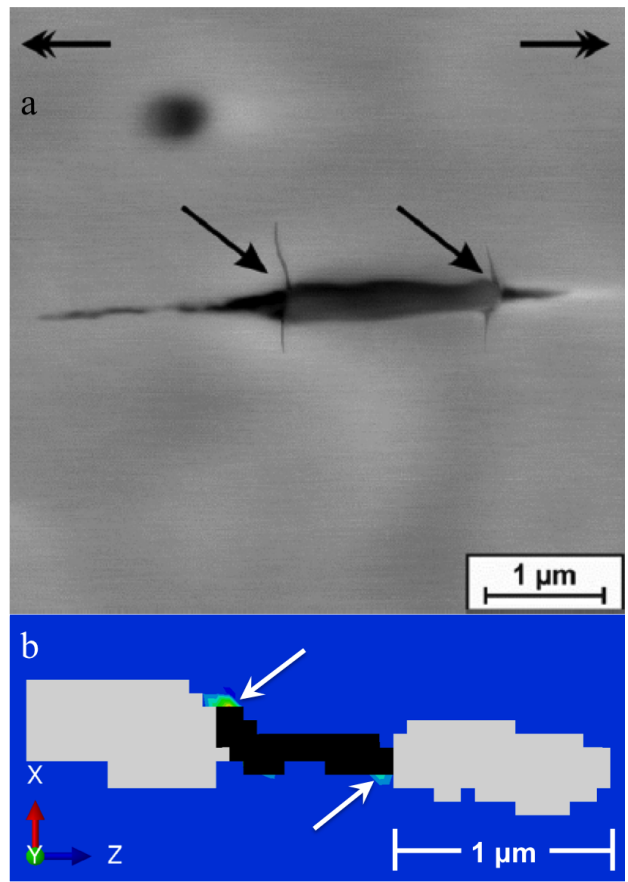

Figure 17: a) SEM of cracks in NiTi at carbide/void interfaces from Ramin et al. [60], used with permission [61] b) equivalent plastic strain contour with inclusions (gray) and void (black), arrows indicate plastic strain hot-spots where peak FIP values occur

Figure 13 and 15 show a three to ten times increase in fatigue life when voiding does not occur. This confirms that it is the debonding and voiding that is increasing the fatigue crack incubation potency around typical inclusion stringers rather than mechanical interaction. Thus, process optimization to reduce voiding and debonding will increase fatigue performance, and stringers formed from fracturing inclusions will be more detrimental than naturally occurring clusters of unfractured inclusions. However, the increase is fatigue life found from removing voiding is still small as compared to the fatigue life improvement caused by increasing NiTi matrix yield strength by $50 \%$ as shown in Figure 14 and 15 . Thus, the three microstructural parameters studied are ranked in order of their influence on fatigue life (where 1 has the highest influence):

1. $50 \%$ increase in matrix yield strength,

2. voiding between inclusions,
3. mechanical interaction between inclusions.

While others have also looked at effects of debonding [11], the results presented in Figure 13 and Figure 15 have the advantage of modeling image-based inclusions rather than an ideal case.

To further justify the rank order above, the increase in fatigue life is studied as a function of fatigue strength for the reconstructed inclusions (two inclusions a with void). The average (over strains $0.27 \%, 0.38 \%$ and $0.5 \%)$ percentage increase in fatigue life $\left(N_{\times}\right)$as a function of matrix yield strength increase $\left(\sigma_{\times}\right)$is shown in Figure 18 , where $N_{\times}=N_{\sigma} / N_{\text {typ }}, \sigma_{\times}=1-\sigma_{y} / \sigma_{y \text {,typ }}$ and $N_{\sigma}$ is the fatigue life at the modified matrix yield strength $\sigma_{y}$, and $N_{\text {typ }}$ is the fatigue life at the typical matrix yield strength $\sigma_{y, \text { typ }}$. From the results in

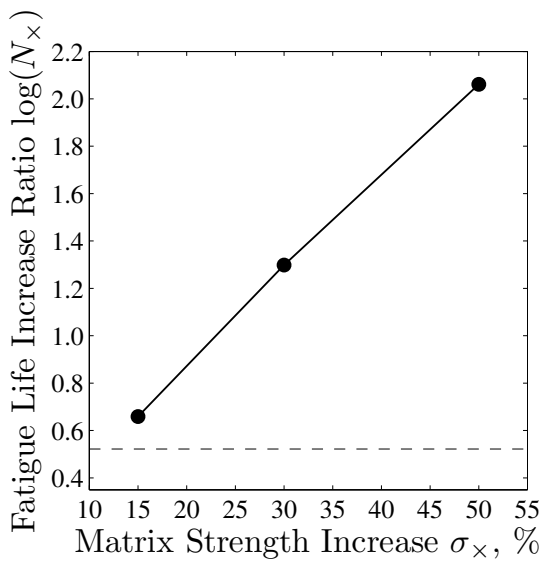

Figure 18: The increase in fatigue life as a function of increase in matrix yield strength for a loading in the $y$-direction

Figure 18, the increase in fatigue life can be parameterized for UHCF strains between $0.27 \%$ and $0.5 \%$ as $\log _{10}\left(N_{\times}\right)=0.040 \sigma_{\times}+0.074$. This expression and Figure 18 show that an increase in matrix yield strength of approximately $11 \%$ would be equivalent to removing inclusion voiding for typical oxicarbide inclusions. The change in fatigue life when the void is removed is shown by the dashed line in Figure 18 for comparison.

\section{Conclusions}

It was shown that inclusions within 1.2 inclusion diameters of the surface have increased potency to incubate fatigue cracks. It was also found that that decreasing applied strain decreased the region of influence of the surface but increased the amplification of fatigue crack incubation potency in such region. The inclusion morphology seemed to control fatigue crack incubation 
more so than inclusion-inclusion interaction, and matrix strengthening was shown to be more effective at increasing fatigue life than removing inclusion-inclusion interactions or voiding. A linear relationship between matrix strengthening and increase in fatigue life (on a semi-log scale) was given for UHCF loadings.

This work presents a simplified approach to complex NiTi systems by focusing only on plastic mechanisms, however with some consideration of dual-phase morphology through elastic calibration. This approach gives a method to isolate the effects of specific microstructural features (i.e., voiding) while controlling others (e.g., grain orientation, dual-phase morphology). The results give evidence supporting a range of process improvements. Surface treatments that reduce crack formation at a depth greater than 1.2 inclusion diameters may be useful for improving fatigue life in $\mathrm{NiTi}$ alloys, and processes such as precipitation strengthening, specially methods that do not lead to stringer formation, may be more effective at improving fatigue performance than processing modifications focused on eliminating stringer formation and/or voiding. The feasibility of processes to change the microstructural features (such as reducing voiding) are not addressed. Rather, this work was intended to gage the relative effect of microstructural changes on fatigue life and to give insight into where efforts could be focused most effectively when designing new alloys. Also, the study of applied strain on surface-inclusion effects gives insight on a mechanical/device design level by outlining a trade-off between decreasing load and increasing fatigue crack incubation potency due to surface effects.

\section{Acknowledgement}

This work was performed under the following financial assistance award 70NANB13H194 from National Institute of Standards and Technology, and 70NANB14H012 from U.S. Department of Commerce, National Institute of Standards and Technology as part of the Center for Hierarchical Materials Design (CHi$\mathrm{MaD})$. This work was also performed, in part, under the auspices of the U.S. Department of Energy by Lawrence Livermore National Laboratory under Contract DEAC52-07NA27344 (LLNL-JRNL-675697). The majority of this work was part of the first author's dissertation [62] and he would like to acknowledge the Predictive Science and Engineering Design (PSED) cluster at Northwestern University, Jacob Smith for his help with high performance computing and for providing the spherical inclusion mesh, and Hongyi Xu for the 3D reconstructions.

\section{References}

[1] A. Toro, F. Zhou, M. H. Wu, W. Van Geertruyden, W. Z. Misiolek, Characterization of non-metallic inclusions in superelastic NiTi tubes, J Mater Eng Perform 18 (5) (2009) 448-458.

[2] A. Pelton, V. Schroeder, M. Mitchell, X.-Y. Gong, M. Barney, S. Robertson, Fatigue and durability of Nitinol stents, J Mech Behav Biomed 1 (2) (2008) 153-164.

[3] T. Nguyen, H. Nguyen, M. Nguyen, S. Komatsu, R. Michiels, Heart valve prosthesis and methods of manufacture and use, US Patent 7,914,569 (Mar. 29 2011).

[4] D. Scheinert, S. Scheinert, J. Sax, C. Piorkowski, S. Bräunlich, M. Ulrich, G. Biamino, A. Schmidt, Prevalence and clinical impact of stent fractures after femoropopliteal stenting, J Am Coll Cardiol 45 (2) (2005) 312-315.

[5] M. Shenoy, R. Kumar, D. McDowell, Modeling effects of nonmetallic inclusions on LCF in DS nickel-base superalloys, Int J Fatigue 27 (2) (2005) 113-127.

[6] R. Kumar, A.-J. Wang, D. McDowell, Effects of microstructure variability on intrinsic fatigue resistance of nickel-base superalloys-a computational micromechanics approach, Int $\mathbf{J}$ Fracture 137 (1-4) (2006) 173-210.

[7] L. Wang, S. Daniewicz, M. Horstemeyer, S. Sintay, A. Rollett, Three-dimensional finite element analysis using crystal plasticity for a parameter study of fatigue crack incubation in a 7075 aluminum alloy, Int J Fatigue 31 (4) (2009) 659-667.

[8] E. S. Alley, R. W. Neu, Microstructure-sensitive modeling of rolling contact fatigue, Int J Fatigue 32 (5) (2010) 841-850.

[9] J. Hochhalter, D. Littlewood, R. Christ Jr, M. Veilleux, J. Bozek, A. Ingraffea, A. Maniatty, A geometric approach to modeling microstructurally small fatigue crack formation: II. physically based modeling of microstructure-dependent slip localization and actuation of the crack nucleation mechanism in AA 7075T651, Model Simul Mater Sc 18 (4) (2010) 045004.

[10] R. Prasannavenkatesan, C. P. Przybyla, N. Salajegheh, D. McDowell, Simulated extreme value fatigue sensitivity to inclusions and pores in martensitic gear steels, Eng Fract Mech 78 (6) (2011) 1140-1155.

[11] N. Salajegheh, D. L. McDowell, Microstructure-sensitive weighted probability approach for modeling surface to bulk transition of high cycle fatigue failures dominated by primary inclusions, Int J Fatigue 59 (2014) 188-199.

[12] X. Wang, B. Xu, Z. Yue, Micromechanical modelling of the effect of plastic deformation on the mechanical behaviour in pseudoelastic shape memory alloys, Int J Plasticity 24 (8) (2008) 1307-1332.

[13] S. Manchiraju, P. Anderson, Coupling between martensitic phase transformations and plasticity: A microstructure-based finite element model, Int J Plasticity 26 (10) (2010) 1508-1526.

[14] S. Manchiraju, D. Gaydosh, O. Benafan, R. Noebe, R. Vaidyanathan, P. M. Anderson, Thermal cycling and isothermal deformation response of polycrystalline NiTi: Simulations vs. experiment, Acta Mater 59 (13) (2011) 5238-5249.

[15] C. Yu, G. Kang, Q. Kan, D. Song, A micromechanical constitutive model based on crystal plasticity for thermo-mechanical cyclic deformation of NiTi shape memory alloys, Int J Plasticity 44 (2013) 161-191.

[16] C. Yu, G. Kang, Q. Kan, Crystal plasticity based constitutive model of NiTi shape memory alloy considering different mechanisms of inelastic deformation, Int J Plasticity 54 (2014) 132162.

[17] C. Yu, G. Kang, Q. Kan, Study on the rate-dependent cyclic deformation of super-elastic NiTi shape memory alloy based on a new crystal plasticity constitutive model, Int J Solids Struct 51 (25) (2014) 4386-4405. 
[18] C. Yu, G. Kang, D. Song, Q. Kan, Effect of martensite reorientation and reorientation-induced plasticity on multiaxial transformation ratchetting of super-elastic NiTi shape memory alloy: New consideration in constitutive model, Int J Plasticity 67 (2015) 69-101.

[19] H. Paranjape, P. M. Anderson, Texture and grain neighborhood effects on Ni-Ti shape memory alloy performance, Model Simul Mater Sc 22 (7) (2014) 075002.

[20] C. Przybyla, R. Prasannavenkatesan, N. Salajegheh, D. L. McDowell, Microstructure-sensitive modeling of high cycle fatigue, Int J Fatigue 32 (3) (2010) 512-525

[21] N. Salajegheh, R. Prasannavenkatesan, D. L. McDowell, G. B. Olson, H.-J. Jou, Finite element simulation of shielding/intensification effects of primary inclusion clusters in high strength steels under fatigue loading, J Eng Mater-T ASME 136 (3) (2014) 031003.

[22] D. L. McDowell, Simulation-based strategies for microstructure-sensitive fatigue modeling, Mat Sci Eng A-Struct 468 (2007) 4-14.

[23] T. Sakai, Review and prospects for current studies on very high cycle fatigue of metallic materials for machine structural use, JSME Int J A-Solid M 3 (3) (2009) 425-439.

[24] E. R. Laskowski MD, What's a normal resting heart rate?, http://www.mayoclinic.org/healthy-living/fitness/expertanswers/heart-rate/faq-20057979, accessed: 2015-01-08.

[25] T. Sakai, Y. Sato, N. Oguma, Characteristic S-N properties of high-carbon-chromium-bearing steel under axial loading in long-life fatigue, Fatigue Fract Eng M 25 (8-9) (2002) 765-773.

[26] M. Chapetti, T. Tagawa, T. Miyata, Ultra-long cycle fatigue of high-strength carbon steels part ii: estimation of fatigue limit for failure from internal inclusions, Mat Sci Eng A-Struct 356 (1) (2003) 236-244.

[27] M. Nakajima, N. Kamiya, H. Itoga, K. Tokaji, H.-N. Ko, Experimental estimation of crack initiation lives and fatigue limit in subsurface fracture of a high carbon chromium steel, Int $\mathbf{J}$ Fatigue 28 (11) (2006) 1540-1546.

[28] D. G. Harlow, R. P. Wei, T. Sakai, N. Oguma, Crack growth based probability modeling of $S-N$ response for high strength steel, Int J Fatigue 28 (11) (2006) 1479-1485.

[29] K. Tosha, D. Ueda, H. Shimoda, S. Shimizu, A study on P-S-N curve for rotating bending fatigue test for bearing steel, Tribol T 51 (2) (2008) 166-172.

[30] T. Sakai, B. Lian, M. Takeda, K. Shiozawa, N. Oguma, Y. Ochi, M. Nakajima, T. Nakamura, Statistical duplex $S-N$ characteristics of high carbon chromium bearing steel in rotating bending in very high cycle regime, Int J Fatigue 32 (3) (2010) 497-504.

[31] S. W. Robertson, M. Launey, O. Shelley, I. Ong, L. Vien, K. Senthilnathan, P. Saffari, S. Schlegel, A. R. Pelton, A statistical approach to understand the role of inclusions on the fatigue resistance of superelastic Nitinol wire and tubing, J Mech Behav Biomed Mater 51 (2015) 119-131.

[32] D. McDowell, K. Gall, M. Horstemeyer, J. Fan, Microstructurebased fatigue modeling of cast A356-T6 alloy, Eng Fract Mech 70 (1) (2003) 49-80.

[33] C.-H. Goh, Crystallographic plasticity in fretting of Ti-6Al-4V, Ph.D. thesis, Georgia Institute of Technology (2002)

[34] A. Fatemi, D. F. Socie, A critical plane approach to multiaxial fatigue damage including out-of-phase loading, Fatigue Fract Eng M 11 (3) (1988) 149-165.

[35] J. Zhang, R. Prasannavenkatesan, M. M. Shenoy, D. L. McDowell, Modeling fatigue crack nucleation at primary inclusions in carburized and shot-peened martensitic steel, Eng Fract Mech 76 (3) (2009) 315-334.

[36] R. McGinty, Multiscale representation of polycrystalline inelasticity, Ph.D. thesis, Georgia Institute of Technology (2001).
[37] R. McGinty, D. McDowell, A semi-implicit integration scheme for rate independent finite crystal plasticity, Int J Plasticity 22 (6) (2006) 996-1025.

[38] T. Belytschko, B. Moran, W. K. Liu, K. Elkhodary, Nonlinear finite element analysis for continua and structures, 2nd Edition, Wiley, 2014.

[39] Y. I. Chumlyakov, N. Surikova, A. Korotaev, Orientation dependence of strength and plasticity of titanium nickelide single crystals, Phys Met Metallogr+ 82 (1) (1996) 102-109.

[40] A. A. Kelly, K. M. Knowles, Crystallography and crystal defects, John Wiley \& Sons, 2012

[41] T. Ezaz, J. Wang, H. Sehitoglu, H. Maier, Plastic deformation of NiTi shape memory alloys, Acta Materialia 61 (1) (2013) 6778.

[42] A. Pelton, G. Huang, P. Moine, R. Sinclair, Effects of thermal cycling on microstructure and properties in nitinol, Materials Science and Engineering: A 532 (2012) 130-138.

[43] K. Otsuka, X. Ren, Recent developments in the research of shape memory alloys, Intermetallics 7 (5) (1999) 511-528.

[44] S. Robertson, A. Pelton, R. Ritchie, Mechanical fatigue and fracture of nitinol, Int Mater Rev 57 (1) (2012) 1-37.

[45] A. Stebner, S. Vogel, R. Noebe, T. Sisneros, B. Clausen, D. Brown, A. Garg, L. Brinson, Micromechanical quantification of elastic, twinning, and slip strain partitioning exhibited by polycrystalline, monoclinic nickel-titanium during large uniaxial deformations measured via in-situ neutron diffraction, Journal of the Mechanics and Physics of Solids 61 (11) (2013) 23022330 .

[46] K. Gall, H. Sehitoglu, R. Anderson, I. Karaman, Y. I Chumlyakov, I. V. Kireeva, On the mechanical behavior of single crystal NiTi shape memory alloys and related polycrystalline phenomenon, Mater Sci Eng A 317 (1) (2001) 85-92.

[47] S. Miyazaki, T. Imai, Y. Igo, K. Otsuka, Effect of cyclic deformation on the pseudoelasticity characteristics of Ti-Ni alloys, Metall Trans A 17 (1) (1986) 115-120.

[48] P. McCormick, Y. Liu, S. Miyazaki, Intrinsic thermalmechanical behaviour associated with the stress-induced martensitic transformation in NiTi, Mater Sci Eng A 167 (1) (1993) 51-56.

[49] S. Miyazaki, K. Mizukoshi, T. Ueki, T. Sakuma, Y. Liu, Fatigue life of $\mathrm{Ti}-50$ at.\% $\mathrm{Ni}$ and $\mathrm{Ti}-40 \mathrm{Ni}-10 \mathrm{Cu}$ (at.\%) shape memory alloy wires, Mater Sci Eng A 273 (1999) 658-663.

[50] K. Gall, H. Maier, Cyclic deformation mechanisms in precipitated NiTi shape memory alloys, Acta Mater 50 (18) (2002) 4643-4657.

[51] K. Gall, J. Tyber, G. Wilkesanders, S. W. Robertson, R. O. Ritchie, H. J. Maier, Effect of microstructure on the fatigue of hot-rolled and cold-drawn NiTi shape memory alloys, Mat Sci Eng A-Struct 486 (1) (2008) 389-403.

[52] A. Pelton, Nitinol fatigue: a review of microstructures and mechanisms, J Mater Eng Perform 20 (4-5) (2011) 613-617.

[53] Y. Kim, S. Miyazaki, Fatigue properties of Ti-50.9 at\% Ni shape memory wires, Proceedings: SMST-97 (1997) 473-477.

[54] P. Adler, J. Allen, J. Lessar, R. Francis, Martensite transformations and fatigue behavior of nitinol, in: Fatigue and Fracture of Medical Metallic Materials and Devices, ASTM International, 2007.

[55] D. Norfleet, P. Sarosi, S. Manchiraju, M.-X. Wagner, M. Uchic, P. Anderson, M. Mills, Transformation-induced plasticity during pseudoelastic deformation in Ni-Ti microcrystals, Acta Materialia 57 (12) (2009) 3549-3561.

[56] H. Sehitoglu, J. Jun, X. Zhang, I. Karaman, Y. Chumlyakov, H. Maier, K. Gall, Shape memory and pseudoelastic behavior of $51.5 \% \mathrm{Ni}-\mathrm{Ti}$ single crystals in solutionized and overaged state, Acta Mater 49 (17) (2001) 3609-3620. 
[57] G. Harlow, Data fusion and science based modeling: A technique for very high cycle fatigue predictions, fourth International Conference on Very High Cycle Fatigue (VHCF-4), Ann Arbor, MI, (2007).

[58] G. Owolabi, R. Prasannavenkatesan, D. McDowell, Probabilistic framework for a microstructure-sensitive fatigue notch factor, Int J Fatigue 32 (8) (2010) 1378-1388.

[59] J. E. Schaffer, D. L. Plumley, Fatigue performance of nitinol round wire with varying cold work reductions, J Mater Eng Perform 18 (5-6) (2009) 563-568.

[60] M. Rahim, J. Frenzel, M. Frotscher, J. Pfetzing-Micklich, R. Steegmüller, M. Wohlschlögel, H. Mughrabi, G. Eggeler, Impurity levels and fatigue lives of pseudoelastic NiTi shape memory alloys, Acta Mater 61 (10) (2013) 3667-3686.

[61] Reprinted from Acta Materialia, 61 (10), M. Rahim, J. Frenzel, M. Frotscher, J. Pfetzing-Micklich, R. Steegmüller, M.Wohlschlögel, H. Mughrabi, G. Eggeler, Impurity levels and fatigue lives of pseudoelastic NiTi shape memory alloys, 36673686, Copyright (2013), with permission from Elsevier.

[62] J. A. Moore, A micromechanics-based method for multiscale fatigue prediction, Ph.D. thesis, Northwestern University (April 2015). 